\title{
A propensity score-matched analysis between patients with high hip dislocation after childhood pyogenic infection and Crowe IV developmental dysplasia of the hip in total hip arthroplasty with subtrochanteric shortening osteotomy
}

\author{
Enze Zhao ${ }^{\dagger} \mathbb{D}$, Zunhan Liư ${ }^{\dagger}$ Zichuan Ding, Zhenyu Luo®, Hao Li and Zongke Zhou* ${ }^{*}$
}

\begin{abstract}
Background: Whether satisfactory clinical and radiological outcomes of total hip arthroplasty (THA) with subtrochanteric shortening osteotomy (SSO) in high hip dislocation after childhood pyogenic infection can be achieved as in Crowe IV developmental dysplasia of the hip (DDH) remains unclear.

Methods: Between September 2009 and December 2016, 151 primary THAs performed at our institution using similar SSO technique and prosthetic design were retrospectively reviewed. After excluding patients who met exclusion criteria, 29 patients were identified as high dislocation (Crowe IV) after childhood infection (HDACl) and 107 as Crowe IV developmental dysplasia of the hip (DDH). Propensity score matching was used to select 29 Crowe IV DDH patients as a control group for the HDACl group with comparable preoperative conditions. Clinical and radiological outcomes and complication were compared and analyzed. The mean follow-up duration of the 2 groups was 5.0 years.

Results: The mean Harris hip score (HHS) and the mean score in range of motion (ROM) domain of the modified Merle d'Aubigné-Postel (MAP) were 84.6 and 4.5 in the HDACl group, compared with 88.3 and 4.9 in the DDH group; there was significant difference between the 2 groups in these parameters ( $P=0.015$ and 0.035 , respectively). Meanwhile, in the HDACl group, the median time of osteotomy union was 4 months and osteotomy nonunion rate was 3\%; no significant difference was detected in the median time of osteotomy union and osteotomy nonunion rate between the 2 groups $(P=0.388$ and 1.000, respectively). And no significant difference was found in the rate of complications between two groups.

(Continued on next page)
\end{abstract}

\footnotetext{
* Correspondence: zhouzongke@scu.edu.cn

'Enze Zhao and Zunhan Liu contributed equally to this work.

Department of Orthopedics, West China Hospital/West China School of Medicine, Sichuan University, 37\# Wuhou Guoxue Road, Chengdu, People's Republic of China
}

C C The Author(s). 2020 Open Access This article is licensed under a Creative Commons Attribution 4.0 International License, which permits use, sharing, adaptation, distribution and reproduction in any medium or format, as long as you give appropriate credit to the original author(s) and the source, provide a link to the Creative Commons licence, and indicate if changes were made. The images or other third party material in this article are included in the article's Creative Commons licence, unless indicated otherwise in a credit line to the material. If material is not included in the article's Creative Commons licence and your intended use is not permitted by statutory regulation or exceeds the permitted use, you will need to obtain permission directly from the copyright holder. To view a copy of this licence, visit http://creativecommons.org/licenses/by/4.0/ The Creative Commons Public Domain Dedication waiver (http://creativecommons.org/publicdomain/zero/1.0/) applies to the data made available in this article, unless otherwise stated in a credit line to the data. 
(Continued from previous page)

Conclusions: HDACl patients who received THA combined with SSO could achieve similar satisfactory results as DDH patients in Crowe type IV. The fixation technique of autogenous cortical bone struts had a positive influence on osteotomy healing of SSO in this specific setting.

Keywords: Total hip arthroplasty, Subtrochanteric shortening osteotomy, High hip dislocation, Childhood pyogenic infection, Developmental dysplasia of the hip

\section{Introduction}

Pyogenic arthritis of the hip is still a common disease for children in less-developed countries [1]. High hip dislocation is a catastrophic sequela of hip pyogenic arthritis [2, 3]. Chronic high hip dislocation could affect patients' normal activities and cause severe pain of hip and low back. Total hip arthroplasty (THA) remains the gold standard for pain relief and hip functional recovery in patients with high dislocation (Crowe IV) after childhood infection (HDACI).

Restoration of the hip rotation center into the true acetabulum in THA has been reported to ensure a more durable prosthesis [4-9]. However, it remains an intractable problem in patients with chronic high hip dislocation. Subtrochanteric shortening osteotomy (SSO) is a useful technique to facilitate the restoration of the anatomic hip rotation center without stretching the nerves, despite pathoanatomy of severely dysplastic hips and extensive contracture in the periarticular soft tissues. Subtrochanteric shortening osteotomy has been reported marked outcomes in THA of Crowe IV DDH [6, 10-12]. However, to our knowledge, limited studies in the literature have reported the results of THA with SSO in patients with high hip dislocation after childhood pyogenic infection [13-18]. Moreover, these prior reports have no control group and include cases without receiving SSO or osteotomy site fixation [13-18].

The objective of this article was to study the result of THA with SSO in the setting of high hip dislocation secondary to childhood pyogenic infection by comparing the clinical and radiological outcomes with Crowe IV DDH patients who underwent THA with SSO. Specifically, we asked (1) whether HDACI patients have similar functional recovery after THA with SSO as Crowe IV DDH patients, (2) whether osteotomy with autogenous cortical bone struts fixation had a satisfied union rate and union time of osteotomy in this specific setting compared with Crowe IV DDH patients, and (3) whether HDACI patients have an increased complication risk compared with Crowe IV DDH patients after THA with SSO.

\section{Materials and methods}

After approval from our institutional review board of West China Hospital, we retrospectively review 151 consecutive patients (160 hips) who underwent primary
THA with SSO between 2009 and 2016. Of these, 5 patients ( 5 hips) died for reasons unrelated to the surgery, and 5 patients ( 5 hips) were lost and could not be contacted by telephone or e-mail. Exclusion criteria removed patients that underwent THA with SSO due to hip pyogenic arthritis secondary to previous hip surgeries (2 hips) or tuberculosis hip infection (3 hips). The remaining cases were identified as $29 \mathrm{HDACI}$ cases and 107 Crowe IV DDH cases with a minimum 3-year follow-up. Hips with evidence of previous pyogenic arthritis, including history (i.e., clear evidence of previous infection of the hip joint from medical or surgical data), the clinical or radiographic features of previous infection (i.e., the old presence of sinus tract), and prior bacteriologic findings, were deemed as the HDACI group. The infecting organism of the original infection (i.e., open incision and drainage in 20 hips and aspirate in 9 hips) was Staphylococcus aureus in 27 hips (93.2\%), Streptococcus in 1 (3.4\%), and Escherichia coli in 1 (3.4\%). Of 29 cases with a history of childhood hip pyogenic infection, all of them were treated with irrigation, debridement, and intravenous antibiotic therapy, and only one patient underwent extra open reduction. The mean age of infection diagnosis was $7.8 \pm 6.3$ years. We support the finding of the earlier reports that the previous infected hips that had remained quiescent for $<10$ years may have increased risk of reactivation of the prior infection after THA and all previous infective hips met the criterion of remained quiescent for $>10$ years with the mean 35 -year quiescent period [19, 20]. According to age, gender, BMI, ASA score, preoperative leg length discrepancy (LLD), and follow-up duration, propensity score matching was used to select $29 \mathrm{DDH}$ patients as a control group (DDH group) for the HDACI group with comparable preoperative conditions. The two groups were compared to study the clinical and radiological outcomes of THA with SSO.

\section{Surgical technique}

All surgeries were designed with transparencies and used cementless femoral and acetabular prosthesis. To restore hip rotation center into the true acetabulum and to improve leg length discrepancy, the amount of femoral shortening was measured and calculated. All legs were 
lengthened by no more than $3-4 \mathrm{~cm}$ to avoid nerve stretching.

All patients received general anesthesia, a posterolateral approach and similar technique of transverse subtrochanteric shortening osteotomy as Yasgur et al. described [21]. After total capsulectomy were performed, intraoperative swab and tissue cultures were conducted due to previous hip pyogenic arthritis and then removal of the femoral head, fibrous scar tissue, and osteophyte were conducted to uncover the true acetabulum. Soft tissue release was performed in all hips. Normally, a transverse femoral osteotomy was conducted approximately $1-2 \mathrm{~cm}$ distal to the lesser trochanter to remove the obstruction of the proximal part of the femur. If proximal femoral canal deformity was presented, SSO was conducted at the proximal deformity. The short section of the vastus lateralis was lifted or split to approach the subtrochanteric region. After transversal osteotomy, the proximal femoral fragment was translated anteriorly to access to the true acetabulum. Then the acetabulum was reamed gradually to prepare the medial wall of the true acetabulum. Press-fit technique was used in all implantation of the acetabular. If there were severe bone defects of acetabulum or insufficient coverage, a structural autograft or titanium alloy (Ti-alloy) mesh combined with bulk bone grafting from the excised femoral head was used.

Then the second transverse subtrochanteric osteotomy was performed to shorten the femur as preoperative planned. The femoral axial intramedullary reaming process was performed sequentially until the appropriate femoral component size was achieved. If a trial hip reduction could not be accomplished after the first cut of osteotomy, the additional transverse osteotomy was gradually conducted to achieve appropriate hip reduction in the true acetabulum. A mean length of subtrochanteric segmental resection was $2.8 \mathrm{~cm}$ (range, 1.8$4.6 \mathrm{~cm}$ ) in the HDACI group and $3.0 \mathrm{~cm}$ (range, 1.9-5.5 $\mathrm{cm}$ ) in the DDH group. The straight stem (S-ROM,
DePuy) was inserted into the femoral intramedullary cavity through the osteotomy site in all patients while ensuring that $15^{\circ}$ to $20^{\circ}$ of anteversion of the femoral component was placed by adjusting rotational alignment of the 2 fragments. In all patients, the longitudinally autogenous cortical bone struts from the resected cylindrical femoral fragment were used to fix the osteotomy site with cables to enhance biologic recovery and autogenous morselized cancellous bone from the resected femoral head was taken to fill the space at the osteotomy site (Figs. 1 and 2). Comparisons of component information and surgical characteristics are summarized in Table 2.

All patients were encouraged to carry out isometric exercises and active limb mobilization on bed immediately after surgery. All patients were allowed partial weight-bearing with crutches as tolerated on the second post-operative day and progressed to full weight-bearing after 6 weeks depending on the stability of the femoral stem and osseous healing at osteotomy site [22].

\section{Clinical evaluations}

Clinical evaluations were conducted preoperatively and 3 weeks, 8 weeks, 16 weeks, and 6 months postoperatively and annually thereafter until the last follow-up. Patients with unusual symptoms or abnormal radiographic findings were conducted with more frequent evaluations. Clinical scores were derived using the Harris hip score (HHS) system, the modified Merle d'Aubigné-Postel (MAP) hip score (including pain, motion, and gait function), and the 12-item short-form health survey (SF-12) [23-25]. In addition to the recognized patient-reported outcomes, all pre- and post-operative evaluations involving Trendelenburg test status were also recorded. The leg length discrepancy (LLD) was measured from the anterior superior iliac spine to the medial malleolus representing the length discrepancy of lower extremities and was recorded pre- and post-operatively. Limb lengthening was defined as the difference in the preoperative and

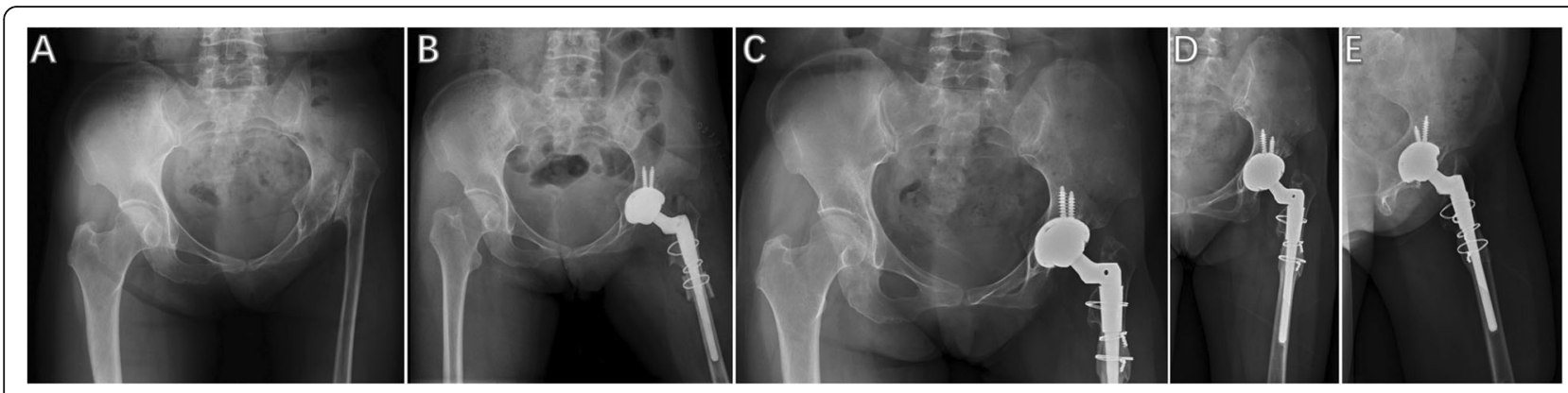

Fig. 1 Radiographs of a 50-year-old female patient with high hip dislocation secondary to childhood pyogenic infection that had a quiescent period of 46 years between infection and left THA. a Preoperative AP pelvic radiograph. $\mathbf{b}$ Immediately postoperative AP pelvic radiograph after left THA with subtrochanteric shortening osteotomy. c Postoperative AP pelvic radiograph 6 years after THA. $\mathbf{d}$ Postoperative AP and e oblique hip radiographs 6 years after THA 


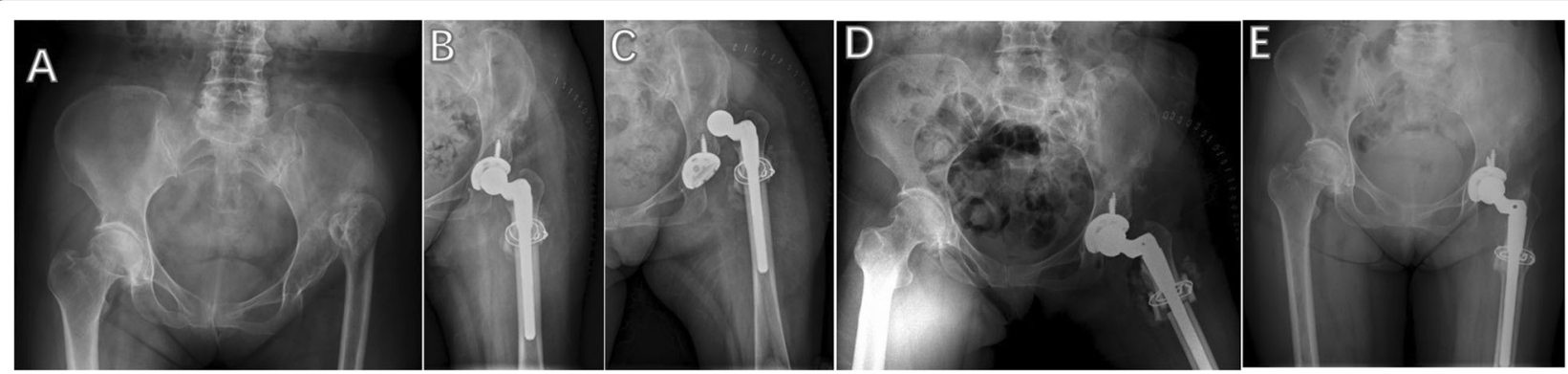

Fig. 2 Radiographs of a 48-year-old female patient with high hip dislocation secondary to childhood pyogenic infection that had a quiescent period of 40 years between infection and left THA. a Preoperative AP pelvic radiograph. $\mathbf{b}$ Immediately postoperative AP hip radiograph. c Dislocation of the left hip 6 days postoperatively. $\mathbf{d}$ Postoperative dislocation treated with closed reduction. e Postoperative AP pelvic radiographs 4 years after THA

postoperative LLD. The individual blood volume was calculated by the Nadler et al. method, and the individual total blood loss was calculated using the difference in hemoglobin levels introduced by the Good et al. [26, 27]. Complications including recurrent infection, periprosthetic fracture, implant instability, nerve palsy, dislocation, nonunion of osteotomy, heterotopic ossification, and revision were also recorded.

\section{Radiographic measurements}

Radiographic analyses were carried out based on serial standardized anteroposterior and lateral radiographs of the affected hip to assess osteotomy healing, osteotomy migration, femoral component stability, component loosening, and heterotopic ossification by two authors who had not participated in the surgery. Periprosthetic radiolucency around acetabulum and femoral components was recorded as described by Delee and Charnley and Gruen et al., respectively [28, 29]. Femoral component loosening was diagnosed by radiographic presence described by Engh et al. [30]. The acetabular component loosening was diagnosed by radiographic presence of progressive radiolucent lines of $>2 \mathrm{~mm}$ around the inserted cup, or migration, or a change in the position of the cup based on the 3 zones around the acetabular cup [31]. Time to osteotomy union was recorded depending

Table 1 Baseline characteristics of all recruited patients

\begin{tabular}{|c|c|c|c|}
\hline Variable & $\mathrm{HDACl}(n=29)$ & $\mathrm{DDH}(n=29)$ & $P$ value \\
\hline Age (years) & $42.8 \pm 10.8$ & $41.7 \pm 12.5$ & 0.720 \\
\hline Female gender & $18(62 \%)$ & $18(62 \%)$ & 1.000 \\
\hline Body mass index $\left(\mathrm{kg} / \mathrm{m}^{2}\right)$ & $22.9 \pm 2.7$ & $22.3 \pm 3.2$ & 0.403 \\
\hline \multicolumn{4}{|l|}{ ASA score } \\
\hline 1 & $26(90 \%)$ & $26(90 \%)$ & 1.000 \\
\hline 2 & $3(10 \%)$ & $3(10 \%)$ & 1.000 \\
\hline Preoperative LLD (cm) & $4.8 \pm 1.7$ & $4.1 \pm 1.6$ & 0.638 \\
\hline Average follow-up (years) & $5.0 \pm 1.8$ & $5.0 \pm 1.8$ & 1.000 \\
\hline Trendelenburg sign (no. of hips) & 29 & 29 & 1.000 \\
\hline Preoperative Harris hip score & $42.9 \pm 9.9$ & $44.1 \pm 9.4$ & 0.654 \\
\hline \multicolumn{4}{|l|}{ Modified MAP } \\
\hline Mean in points (mean \pm SD) & $6.5 \pm 2.0$ & $6.6 \pm 2.1$ & 0.949 \\
\hline Pain & $2.1 \pm 0.9$ & $2.2 \pm 0.8$ & 0.649 \\
\hline Walking & $1.9 \pm 0.5$ & $1.8 \pm 0.5$ & 0.612 \\
\hline $\mathrm{ROM}$ & $2.5 \pm 0.9$ & $2.5 \pm 0.9$ & 1.000 \\
\hline \multicolumn{4}{|l|}{ SF-12 } \\
\hline PCS & $10.5 \pm 1.5$ & $10.8 \pm 1.6$ & 0.451 \\
\hline MCS & $13.7 \pm 1.9$ & $14.1 \pm 1.4$ & 0.347 \\
\hline
\end{tabular}

HDACI high dislocation after childhood infection, DDH developmental dysplasia of the hip, ASA American Society of Anesthesiologists, LLD leg length discrepancy, MAP Merle d'Aubigné-Postel, ROM range of motion, SF-12 12-item short-form health survey questionnaire, MCS mental component summary, PCS physical component summary, SD standard deviation 
on radiographic presence described by Masonis et al. [32]. The heterotopic ossification was assessed by using the method described by Brooker et al. [33]. Subsidence of the femoral component was assessed as the alteration in the distance from the center of the superomedial tip of the femoral stem to the most proximal point on the lesser trochanter and the change of $>5 \mathrm{~mm}$ was considered meaningful. Osteointegration of the femoral prosthesis was classified (i.e., bone ingrown, stable fibrous ingrown, or unstable) as described by Engh et al. [34].

\section{Statistical analysis}

Propensity scores depending on logistic regression was used to match the HDACI group and DDH group. Student's $t$ test and the Wilcoxon rank-sum test were used to analyzed continuous variables while comparing between two groups. The paired $t$ test was used to analyze the preoperative and postoperative continuous variables for each group. Categorical values were analyzed using the chi-squared or Fisher's exact test. All statistical analyses were performed using SPSS v25.0 (IBM, Armonk, NY). $P$ values $<0.05$ were considered statistically significant.

\section{Results}

The comparisons of the demographic data between two groups are summarized in Table 1; there were no significant differences between the 2 groups before the surgery. The mean preoperative LLD was $4.8 \mathrm{~cm}$ in the HDACI group and $4.1 \mathrm{~cm}$ in the DDH group $(P=$ 0.638). All patients had preoperative whole blood cell counts, C-reactive protein limits, erythrocyte sedimentation rates, and IL- 6 levels that were within the normal ranges. All intraoperative examinations were found to be negative for infection including intraoperatively frozen sections, synovial fluid, synovial tissues, and excised specimen cultures. No significant difference was found in perioperative blood loss and operation time between the 2 groups (Table 2).

The postoperative clinical outcomes are summarized in Table 3. The mean Harris hip score, modified MAP score, SF-12 score, and Trendelenburg sign significantly improved postoperatively compared with preoperatively in each group. At the last follow-up, the mean postoperative HHS was 84.6 in the HDACI group and 88.3 in the DDH group $(P=0.015)$. Although no significant difference was seen between the 2 groups in the mean modified MAP score $(P=0.149)$, there was significant difference between the 2 groups in the mean score in range of motion domain of the modified MAP with 4.5 in the HDACI group and 4.9 in the DDH group $(P=$ 0.035). The mean postoperative LLD was $1.2 \mathrm{~cm}$ in the HDACI group and $1.0 \mathrm{~cm}$ in the DDH group $(P=$ $0.459)$.
Table 2 Component information and surgical characteristics

\begin{tabular}{|c|c|c|c|}
\hline Variable & $\begin{array}{l}\text { HDACl }(n= \\
29)\end{array}$ & $\begin{array}{l}\mathrm{DDH}(n= \\
29)\end{array}$ & $\begin{array}{l}P \\
\text { value }\end{array}$ \\
\hline Cup diameter (mm) & & & 0.793 \\
\hline 38 & 1 & 0 & \\
\hline 40 & 3 & 2 & \\
\hline 42 & 1 & 2 & \\
\hline 44 & 12 & 12 & \\
\hline 46 & 3 & 7 & \\
\hline 48 & 6 & 4 & \\
\hline 50 & 0 & 0 & \\
\hline 52 & 3 & 2 & \\
\hline Head diameter (mm) & & & 0.758 \\
\hline 22 & 5 & 5 & \\
\hline 28 & 15 & 18 & \\
\hline 32 & 6 & 5 & \\
\hline 36 & 3 & 1 & \\
\hline $\begin{array}{l}\text { Types of bearing surface (no. of } \\
\text { hips) }\end{array}$ & & & 0.913 \\
\hline Ceramic-on-ceramic bearing & 18 & 18 & \\
\hline $\begin{array}{l}\text { Ceramic-on-polyethylene } \\
\text { bearing }\end{array}$ & 5 & 6 & \\
\hline $\begin{array}{l}\text { Metal-on-polyethylene } \\
\text { bearing }\end{array}$ & 6 & 5 & \\
\hline Length of femoral resection $(\mathrm{cm})$ & $2.8 \pm 0.9$ & $3.0 \pm 1.2$ & 0.396 \\
\hline Operation time (min) & $157.6 \pm 24.2$ & $146.1 \pm 27.3$ & 0.095 \\
\hline Perioperative blood loss (mL) & $457 \pm 170$ & $382 \pm 166$ & 0.094 \\
\hline
\end{tabular}

$H D A C l$ high dislocation after childhood infection, $D D H$ developmental dysplasia of the hip

The radiographic results are summarized in Table 4 . There was no significant difference in the median time for union of the osteotomy site between two groups (median 4 vs 4 months, $P=0.388$ ). At the latest followup, in the HDACI group, a nonprogressive radiolucent line $(<2 \mathrm{~mm})$ was seen in 2 hips around the acetabular cup ( 1 in zone I and 1 in zone II, respectively) and in 2 hips around the femoral stem ( 1 in zone 1 and 1 in zone 7). And a progressive radiolucent line was seen in one hip around undersized femoral stem of the HDACI group with nonunion of the osteotomy site, aseptic loosening, stem subsidence, and femoral stem breakage. Stem revision was performed with a larger monoblock cementless femoral stem 1 year after the index arthroplasty. In the DDH group, there were 2 hips presenting nonprogressive radiolucent line around the acetabular cup ( 2 in zone II) and 2 hips presenting nonprogressive radiolucent line around the femoral stem ( 1 in zone 1 and 1 in zone 7). There was no significant difference between the 2 groups in stem stability according to the Engh classification $(P=0.670)$ [34]. In comparison of 
Table 3 Postoperative clinical outcomes

\begin{tabular}{|c|c|c|c|}
\hline Variable & $\mathrm{HDACl}(n=29)$ & $\mathrm{DDH}(n=29)$ & $P$ value \\
\hline Postoperative LLD (cm) & $1.2 \pm 0.9$ & $1.0 \pm 0.7$ & 0.459 \\
\hline Trendelenburg sign (no. of hips) & 2 & 0 & 0.491 \\
\hline Postoperative Harris hip score & $84.6 \pm 6.2$ & $88.3 \pm 5.2$ & 0.015 \\
\hline \multicolumn{4}{|l|}{ Postoperative modified MAP } \\
\hline Mean in points (mean \pm SD) & $14.7 \pm 1.6$ & $15.3 \pm 1.6$ & 0.149 \\
\hline Pain & $5.7 \pm 0.5$ & $5.7 \pm 0.6$ & 0.821 \\
\hline Walking & $4.6 \pm 0.6$ & $4.8 \pm 0.6$ & 0.147 \\
\hline ROM & $4.5 \pm 0.6$ & $4.9 \pm 0.6$ & 0.035 \\
\hline \multicolumn{4}{|l|}{ SF-12 } \\
\hline PCS & $20.3 \pm 1.6$ & $20.9 \pm 1.5$ & 0.125 \\
\hline MCS & $23.5 \pm 1.7$ & $24.1 \pm 1.8$ & 0.171 \\
\hline
\end{tabular}

the two groups, heterotopic ossification was not significantly different (6 in the HDACI group and 4 in DDH group, $P=0.487$ ) on the last follow-up radiographs. According to the Brooker classification system asymptomatic class-I heterotopic ossification was observed in 4 hips of the HDACI group and 3 hips of the DDH group and class-II was observed in 2 hips of the HDACI group and one hip of the DDH group.

No periprosthetic infection was observed during the follow-up in each group (Table 5). Two hips underwent surgical site infection in the HDACI group and recovered after debridement, intravenous, and oral antibiotic therapy. There was one femoral nerve palsy combined with sciatic nerve palsy and 2 femoral nerve palsies in the HDACI group, and one sciatic nerve palsy in the DDH group, all of which recovered fully after neurotrophic therapy and rehabilitation training within 6 months without further sequelae. Seven hips in the HDACI group and six hips in DDH group occurred

Table 4 Radiographic Outcomes

\begin{tabular}{llll}
\hline Parameters & HDACl $(\boldsymbol{n}=\mathbf{2 9})$ & DDH $(\boldsymbol{n}=\mathbf{2 9})$ & $\boldsymbol{P}$ value \\
\hline Time for union of SSO (mo) & $4(3-10)^{\mathrm{b}}$ & $4(2-10)$ & 0.388 \\
Radiolucency around cup & $2(7 \%)$ & $2(7 \%)$ & 1.000 \\
Radiolucency around stem & $3(10 \%)$ & $2(7 \%)$ & 1.000 \\
Stem subsidence & $1(3 \%)$ & $0(0 \%)$ & 1.000 \\
Stem stability & & & 0.670 \\
$\quad$ Bone ingrowth & $25(87 \%)$ & $27(93 \%)$ & \\
$\quad$ Fibrous stable & $3(10 \%)$ & $2(7 \%)$ & \\
$\quad$ Loosening & $1(3 \%)$ & $0(0 \%)$ & \\
Heterotopic ossification & $6(21 \%)$ & $4(14 \%)$ & 0.487
\end{tabular}

HDACI high dislocation after childhood infection, $D D H$ developmental dysplasia of the hip, SSO subtrochanteric shortening osteotomy ${ }^{a}$ The values are given as the median with the range in parentheses

${ }^{b}$ One outliner is removed from the data intraoperative femoral fracture, all of which were fixed with cerclage cable. Two patients in the HDACI group and one patient in the DDH group experienced postoperative dislocation, which was treated with closed reduction (Fig. 2). In each group, there was one patient underwent postoperative dislocation with treatment of open reduction without additional recurrence. As aforementioned, there was one patient in the HDACI group with nonunion of the osteotomy site, aseptic loosening, and femoral stem crack. Femoral revisions were performed in the patient and showed excellent outcome at last follow-up.

\section{Discussion}

The restoration of the hip rotation center into the true acetabulum promotes long-term durability with lower loosening and aseptic revision rates in patients who received THA [35]. In THA for severe DDH, SSO has been shown to facilitate restoration of the anatomical hip rotation center and provide excellent exposure of the true acetabulum [36]. However, for patients with high hip dislocation after childhood pyogenic infection, limited studies have reported series of who received THA and SSO using a single method to fix the osteotomy site [13, 15-17]. Furthermore, to our knowledge, only one study has compared the results of THA and SSO with multiple fixation between these 2 etiologies [14]. Therefore, we attempt to conduct the present study by comparing the clinical and radiographic outcomes of THA and transverse SSO with autogenous cortical bone struts fixation between HDACI and Crowe IV DDH patients.

The challenge of THA in chronic dislocation depends on the severity of anatomic deformation including high hip center, small femoral canal, rotational deformity of the proximal femur, thickening of the capsule, dysfunction of the transverse abductor, thickening of the 
Table 5 Complications

\begin{tabular}{llll}
\hline Complications & HDACl $(\boldsymbol{n}=\mathbf{2 9})$ & DDH $(\boldsymbol{n}=\mathbf{2 9})$ & $\boldsymbol{P}$ value \\
\hline Surgical site infection & $2(7 \%)$ & $0(0 \%)$ & 0.491 \\
Periprosthetic infection & $0(0 \%)$ & $0(0 \%)$ & 1.000 \\
Sciatic nerve palsy & $1(3 \%)$ & $1(3 \%)$ & 1.000 \\
Femoral nerve palsy & $3(10 \%)$ & $0(0 \%)$ & 0.237 \\
Intraoperative periprosthetic femoral fracture & $7(24 \%)$ & $6(21 \%)$ & 0.753 \\
Dislocation & $3(10 \%)$ & $2(7 \%)$ & 1.000 \\
Aseptic loosening & $1(3 \%)$ & $0(0 \%)$ & 1.000 \\
Osteotomy site nonunion & $1(3 \%)$ & $0(0 \%)$ & 1.000 \\
\hline
\end{tabular}

HDACI high dislocation after childhood infection, $D D H$ developmental dysplasia of the hip

iliopsoas, contracture of the hamstring, adductor and rectus femoris muscles, and contracture of the sciatic nerve and deep femoral artery, which also influence the postoperative results $[3,37]$. We found that the mean postoperative Harris hip score and modified MAP score in our HDACI group are comparable with prior studies [13-17]. However, lower mean score in postoperative Harris hip score and range of motion domain of the modified MAP was identified in the HDACI group compared with the DDH group in the present study. Similar results have been reported in several literatures [14, 15]. The possible interpretations may include the following: first, besides aforementioned anatomic deformation of chronic dislocation, previous infection may result in more severe soft tissue contractures, changed positions of the femoral nerve and vessels, and fibrotic muscles; second, a number of scar tissue repair after previous surgical procedures led to limited range of motion; and third, to avoid damaging deformed neurovascular structures and enhance recovery after surgery, we choose limited soft tissue release rather than widely exposure as far as possible during the index surgery.

In this study, we used similar technique in all groups, including fixation of osteotomy site with cables and the longitudinally split removed autogenous segment. An important finding of this study is that SSO with autogenous cortical bone struts fixation has a predictable and high rate of osteotomy healing within a relatively rapid time in the setting of HDACI. The nonunion rate of SSO in both groups is comparable with previous studies of DDH patients reporting a nonunion rate that ranged from 0 to $11 \%[21,38,39]$. There was only 1 case of osteotomy nonunion in the HDACI group, which healed successfully after stem reimplantation surgery with satisfying outcomes during the follow-up. In the remaining 28 HDACI patients, osteotomy healing occurred at a median time of 4 months, comparable with that in DDH group. Park et al. [14] compared the results of THA with SSO between 25 HDACI patients and 25 Crowe IV DDH patients and reported longer median time for union of SSO and higher reoperation rate of additional fixation in the HDACI patients. However, $40 \%$ and 32\% HDACI patients were performed with non-fixed SSO and additional longitudinal osteotomy, respectively, which may confound their results. Using plate and screw for fixation of transverse osteotomy were also reported and showed satisfying outcomes $[6$, 21]. Nevertheless, the periosteal damage may have a delaying effect on osteotomy healing while applying a plate and screw. Catma et al. reported that fixation of the longitudinally split removed autogenous segment with cables may provide competent rotational stability and resulted in early union at the osteotomy site [40]. Therefore, although the S-ROM prosthesis can serve as an intramedullary nail splinting the osteotomy site and SSO without any fixation was reported with satisfying outcomes in DDH patients $[5,41]$, we recommend routinely using autogenous cortical bone struts on the site of SSO with cerclage wiring in HDACI patients who underwent THA and SSO.

Concerns of increased the complications rate and recurrent infection always exist when reconstructing hips with previous hip septic arthritis. Kim et al. reported that the previous infected hips that had remained quiescent for $<10$ years may have increased risk of reactivation of the prior infection after THA $[19,20]$. In the present study, all previous infective hips met the criterion of over 10-year quiescent period without positive finding for evidence of active infection and no recurrent infection happened during the follow-up. However, there are two cases in the HDACI group who experienced surgical site infection with different infecting organism from the original infection after the index surgery. One of the cases, a 44-year-old male, had hip joint infection (Staphylococcus aureus) remained quiescent for 40 years at the time of THA and SSO. Three years after the index surgery, a subcutaneous abscess (Nontuberculous mycobacteria) appeared in the surgical site. Fortunately, the abscess did not communicate with the articular cavity and the infection did not recur after treatment with focal 
debridement, intravenous, and oral antibiotic therapy. Therefore, bacteriological samples should be collected extensively to maximize the opportunities of discovering residual bacteria in order to prevent catastrophic consequences such as periprosthetic joint infection among these susceptible patients with a history of childhood pyogenic infection.

Higher rates of postoperative complications in the HDACI group were mostly caused by nerve palsy and dislocation rather than reactivation of the prior infection. It was reported that the risk of neuralgic traction injury increases when restoration of the anatomic hip rotation center requires limb lengthening in excess of 3-4 $\mathrm{cm}[42,43]$. In the present study, 3 patients $(10 \%)$ in the HDACI group experienced temporary femoral nerve paralysis postoperatively without further sequelae and the mean leg lengthening of the three was $2.7 \mathrm{~cm}$. Although both groups had overall low incidence of nerve palsy, there was a trend toward a higher rate in the HDACI group. Due to contracture and adhesions in the HDACI group, the nerve extensibility is worse than that in Crowe IV DDH patients so that the safe range of limb lengthening for non-pyogenic DDH patients may also cause nerve injury in HDACI patients. Therefore, care should be taken to conduct meticulous surgical manipulation including proper retractor placement, technique of effective soft tissue release, and relatively less limb lengthening in this group of patients.

Several limitations exist in the present study. First, the retrospective nature of the present study decreased the level of evidence. Second, sample size was relatively small. Third, mean 5-year follow-up is relatively shortterm, and longer-term follow-up and larger sample size would provide more meaningful data. Fourth, the operations were conducted by 5 senior joint surgeons; therefore, the differences in surgical manipulations between surgeons may cause potential statistical bias. Despite these limitations, this study involved the relatively large of such sample sizes to date comparing the clinical and radiological outcomes of THA with SSO between the HDACI and Crowe IV DDH, two uncommon diagnoses. In addition, different surgeons made the study results easier to replicate and generalize. The importance of this study is that it demonstrated the efficacy and safety of SSO with predictable osteotomy healing in the setting of high hip dislocation after childhood pyogenic infection.

\section{Conclusions}

Although HDACI patients who received THA combined with SSO had relatively lower clinical scores compared with Crowe IV DDH patients, satisfactory results could also be achieved in HDACI patients. Furthermore, our study also suggests that the fixation technique of autogenous cortical bone struts may enhance the union of osteotomy site in HDACI patients who received THA and SSO. Therefore, THA and SSO combined with the fixation technique of autogenous cortical bone struts remains a good choice in HDACI patients, not at the cost of increasing the rate of overall complications and the risk of recurrent infection.

\section{Abbreviations}

DDH: Developmental dysplasia of the hip; HDACl: High dislocation after childhood infection; ASA: American Society of Anesthesiologists; LLD: Leg length discrepancy; MAP: Merle d'Aubigné-Postel; ROM: Range of motion; SF12: 12-item short-form health survey questionnaire; MCS: Mental component summary; PCS: Physical component summary; SD: Standard deviation

\section{Acknowledgements}

Not applicable

\section{Authors' contributions}

EZ-Z and ZH-L performed data collection and analysis. ZC-D, ZY-L, and H-L performed manuscript writing. ZK-Z drafted the work. The authors read and approved the final manuscript.

\section{Funding}

This work was supported by Key Research \& Development program of Science \& Technology Department of Sichuan Province (No.2018SZ0135 and 2018SZ0255). The funders had no role in study design, data collection, data analysis, decision to publish, or preparation of the manuscript.

\section{Availability of data and materials}

The datasets analyzed during the current study are available from the corresponding author on reasonable request.

\section{Ethics approval and consent to participate}

This retrospective study was approved by the Institutional Review Board of West China Hospital, Sichuan University (ID number: 2012-268). All patients provided informed consent for participation.

Consent for publication

Not applicable

\section{Competing interests}

The authors declare that they have no competing interests

Received: 15 January 2020 Accepted: 7 September 2020

Published online: 17 September 2020

References

1. Nunn TR, Cheung WY, Rollinson PD. A prospective study of pyogenic sepsis of the hip in childhood. J Bone Joint Surg Br. 2007;89(1):100-6.

2. Chen CE, Wang JW, Juhn RJ. Total hip arthroplasty for primary septic arthritis of the hip in adults. Int Orthop. 2008:32(5):573-80.

3. Kim YH, Oh SH, Kim JS. Total hip arthroplasty in adult patients who had childhood infection of the hip. J Bone Joint Surg Am Vol. 2003;85a(2):198-204.

4. Ahmed E, el Ibrahim G, Ayman B. Total hip arthroplasty with subtrochanteric osteotomy in neglected dysplastic hip. Int Orthop. 2015; 39(1):27-33

5. Reikeraas $\mathrm{O}$, et al. Femoral shortening in total arthroplasty for completely dislocated hips: 3-7 year results in 25 cases. Acta Orthop Scand. 1996;67(1): 33-6.

6. Bruce WJ, et al. A new technique of subtrochanteric shortening in total hip arthroplasty: surgical technique and results of 9 cases. J Arthroplasty. 2000; 15(5):617-26.

7. Hartofilakidis G, Stamos K, loannidis TT. Low friction arthroplasty for old untreated congenital dislocation of the hip. J Bone Joint Surg Br. 1988;70(2): 182-6.

8. Linde F, Jensen J. Socket loosening in arthroplasty for congenital dislocation of the hip. Acta Orthop Scand. 1988;59(3):254-7.

9. Paavilainen T, Hoikka V Solonen KA. Cementless total replacement for severely dysplastic or dislocated hips. J Bone Joint Surg Br. 1990;72(2):205-11. 
10. Wang $D$, et al. Long-term results of cementless total hip arthroplasty with subtrochanteric shortening osteotomy in Crowe type IV developmental dysplasia. J Arthroplasty. 2017;32(4):1211-9.

11. Wang $D$, et al. Subtrochanteric shortening osteotomy during cementless total hip arthroplasty in young patients with severe developmental dysplasia of the hip. BMC Musculoskelet Disord. 2017:18(1):491.

12. Krych AJ, et al. Total hip arthroplasty with shortening subtrochanteric osteotomy in Crowe type-IV developmental dysplasia: surgical technique. J Bone Joint Surg Am. 2010;92(Suppl 1 Pt 2):176-87.

13. Yang $Y$, et al. Outcomes after total hip arthroplasty using a cementless SROM modular stem for patients with high hip dislocation secondary to hip pyogenic arthritis. Orthop Surg. 2019;11(3):460-6.

14. Park CW, et al. Total hip arthroplasty with subtrochanteric shortening osteotomy in patients with high hip dislocation secondary to childhood septic arthritis: a matched comparative study with Crowe IV developmental dysplasia. J Arthroplasty. 2020;35(1):204-11.

15. Kim YH, Seo HS, Kim JS. Outcomes after THA in patients with high hip dislocation after childhood sepsis. Clin Orthop Relat Res. 2009;467(9):2371-8.

16. Zeng WN, et al. Midterm results of total hip arthroplasty in patients with high hip dislocation after suppurative hip arthritis. J Arthroplasty. 2019;34(1): 102-7.

17. Wang D, et al. Long-term results of cementless total hip arthroplasty for patients with high hip dislocation after childhood pyogenic infection. J Arthroplasty. 2019;34(10):2420-6.

18. Luo $Y$, et al. Clinical outcomes and quality of life after total hip arthroplasty in adult patients with a history of infection of the hip in childhood: a midterm follow-up study. J Orthop Surg Res. 2019;14(1):38.

19. Kim YH, Kim JS. Total hip arthroplasty in adult patients who had developmental dysplasia of the hip. J Arthroplasty. 2005;20(8):1029-36

20. Kim YH. Total arthroplasty of the hip after childhood sepsis. J Bone Joint Surg Br Vol. 1991;73(5):783-6.

21. Yasgur DJ, et al. Subtrochanteric femoral shortening osteotomy in total hip arthroplasty for high-riding developmental dislocation of the hip. J Arthroplasty. 1997;12(8):880-8.

22. Ding ZC, et al. Limited influence of comorbidities on length of stay after total hip arthroplasty: experience of enhanced recovery after surgery. Orthop Surg. 2020;12(1):153-61.

23. Harris WH. Traumatic arthritis of the hip after dislocation and acetabular fractures: treatment by mold arthroplasty. An end-result study using a new method of result evaluation. J Bone Joint Surg Am. 1969;51(4):737-55.

24. Ovre S, et al. Comparison of distribution, agreement and correlation between the original and modified Merle d'Aubigne-Postel Score and the Harris Hip Score after acetabular fracture treatment: moderate agreement, high ceiling effect and excellent correlation in 450 patients. Acta Orthop. 2005;76(6):796-802.

25. Ware J, Kosinski M, Keller SD. A 12-Item Short-Form Health Survey: construction of scales and preliminary tests of reliability and validity. Med Care. 1996;34(3):220-33.

26. Nadler SB, Hidalgo JH, Bloch T. Prediction of blood volume in normal human adults. Surgery. 1962;51(2):224-32.

27. Good L, Peterson E, Lisander B. Tranexamic acid decreases external blood loss but not hidden blood loss in total knee replacement. Br J Anaesth. 2003;90(5):596-9.

28. DeLee JG, Charnley J. Radiological demarcation of cemented sockets in total hip replacement. Clin Orthop Relat Res. 1976;121:20-32.

29. Gruen TA, McNeice GM, Amstutz HC. "Modes of failure" of cemented stemtype femoral components: a radiographic analysis of loosening. Clin Orthop Relat Res. 1979;141:17-27.

30. Engh CA, Massin P, Suthers KE. Roentgenographic assessment of the biologic fixation of porous-surfaced femoral components. Clin Orthop Relat Res. 1990;257:107-28.

31. Fredin $\mathrm{H}$, et al. Total hip arthroplasty in high congenital dislocation. 21 hips with a minimum five-year follow-up. J Bone Joint Surg Br. 1991;73(3):430-3.

32. Masonis JL, et al. Subtrochanteric shortening and derotational osteotomy in primary total hip arthroplasty for patients with severe hip dysplasia: 5-year follow-up. J Arthroplasty. 2003;18(3 Suppl 1):68-73.

33. Brooker AF, et al. Ectopic ossification following total hip replacement. Incidence and a method of classification. J Bone Joint Surg Am. 1973;55(8): 1629-32.

34. Engh CA, Glassman AH, Suthers KE. The case for porous-coated hip implants. The femoral side. Clin Orthop Relat Res. 1990;261:63-81.
35. Becker DA, Gustilo RB. Double-chevron subtrochanteric shortening derotational femoral osteotomy combined with total hip arthroplasty for the treatment of complete congenital dislocation of the hip in the adult. Preliminary report and description of a new surgical technique. J Arthroplasty. 1995;10(3):313-8.

36. Sofu $\mathrm{H}$, et al. Transverse subtrochanteric shortening osteotomy during cementless total hip arthroplasty in Crowe type-III or IV developmental dysplasia. J Arthroplasty. 2015;30(6):1019-23.

37. Wu $X_{\text {, et }}$ al. The techniques of soft tissue release and true socket reconstruction in total hip arthroplasty for patients with severe developmental dysplasia of the hip. Int Orthop. 2012;36(9):1795-801.

38. Oe K, et al. Subtrochanteric shortening osteotomy combined with cemented total hip arthroplasty for Crowe group IV hips. Arch Orthop Trauma Surg. 2013;133(12):1763-70.

39. Kiliçoğlu $\mathrm{O}$, et al. Cementless total hip arthroplasty with modified oblique femoral shortening osteotomy in Crowe type IV congenital hip dislocation. J Arthroplasty. 2013;28(1):117-25.

40. Catma MF, et al. Femoral shortening osteotomy in total hip arthroplasty for severe dysplasia: a comparison of two fixation techniques. Int Orthop. 2016; 40(11):2271-6.

41. Onodera S, et al. Cementless total hip arthroplasty using the modular SROM prosthesis combined with corrective proximal femoral osteotomy. J Arthroplasty. 2006;21(5):664-9.

42. Crowe JF, Mani VJ, Ranawat CS. Total hip replacement in congenital dislocation and dysplasia of the hip. J Bone Joint Surg Am. 1979;61(1):15-23.

43. Anwar MM, et al. Total hip arthroplasty in the neglected congenital dislocation of the hip. A five- to 14-year follow-up study. Clin Orthop Relat Res. 1993;295:127-34.

\section{Publisher's Note}

Springer Nature remains neutral with regard to jurisdictional claims in published maps and institutional affiliations.

Ready to submit your research? Choose BMC and benefit from:

- fast, convenient online submission

- thorough peer review by experienced researchers in your field

- rapid publication on acceptance

- support for research data, including large and complex data types

- gold Open Access which fosters wider collaboration and increased citations

- maximum visibility for your research: over $100 \mathrm{M}$ website views per year

At $\mathrm{BMC}$, research is always in progress.

Learn more biomedcentral.com/submissions 